\title{
Questes
}

Revue pluridisciplinaire d'études médiévales

\section{Le roi, le tyran et le sage : Charles VI, Evilmerodag et Moïse dans Le Songe du vieil pelerin}

\section{Amandine Mussou}

\section{CpenEdition}

\section{Journals}

Édition électronique

URL : http://journals.openedition.org/questes/1504

DOI : 10.4000/questes. 1504

ISSN : 2109-9472

\section{Éditeur}

Les Amis de Questes

\section{Édition imprimée}

Date de publication : 15 janvier 2008

Pagination : 67-80

ISSN : 2102-7188

\section{Référence électronique}

Amandine Mussou, « Le roi, le tyran et le sage : Charles VI, Evilmerodag et Moïse dans Le Songe du vieil pelerin », Questes [En ligne], 13 | 2008, mis en ligne le 01 janvier 2014, consulté le 01 mai 2019. URL http://journals.openedition.org/questes/1504; DOI : 10.4000/questes.1504 


\title{
Le roi, le tyran et le sage : Charles VI, Evilmerodag et Moïse dans Le Songe du vieil pelerin
}

Amandine Mussou

\begin{abstract}
À la fin de l'année 1388, Charles VI est déclaré majeur et rappelle près de lui les anciens conseillers de son père, jusque-là évincés par ses propres oncles. L'année suivante, Philippe de Mézières accomplit un acte politique fort $^{1}$ en achevant la rédaction du Songe du vieil pelerin. Depuis 1380 et la mort de Charles V, dont il était un proche, il s'était retiré dans le couvent des Célestins à Paris. C'est là qu'il compose en langue vernaculaire le long récit allégorique du Songe du vieil pelerin destiné à Charles $\mathrm{VI}^{2}$. Philippe de Mézières, chargé par Charles V de l'éducation du dauphin, place tous ses espoirs de réforme dans la personne du jeune roi désormais aux commandes de l'État.

L'auteur poursuit le projet chimérique de la reprise d'une croisade générale en Europe pour la restauration de l'Empire latin de Constantinople et la reconquête des lieux saints. Ce projet politique et idéologique repose sur la mise en place d'un personnel allégorique. Dans le prologue, Divine Providence apparaît en songe au vieil pelerin, le narrateur, incarné dans le récit par deux
\end{abstract}

\footnotetext{
${ }^{1}$ Voir sur ce point Pierre-Yves BADEL, Le Roman de la rose au XIV siècle. Étude de la réception de l'œuvre, Genève, Droz, 1980, p. 382 sqq.

${ }^{2}$ «Cy commaince l'aucteur son livre intitule le Songe du Vieil Pelerin, adroissant au Blanc Faucon au bec et piez dorez (...) », PhiLIPPE DE MÉzIÈres, Le Songe du Vieil Pelerin, George William Coopland (éd.), Cambridge, Cambridge University Press, 1969, t. 1, p. 190.
} 
personnages, Ardant Désir et sa sœur Bonne Espérance ${ }^{3}$. Divine Providence lui annonce qu'il va partir à la recherche des Reines Charité, Sapience et Vérité afin de les ramener sur terre pour réformer la Chrétienté. L'ouvrage retrace dans le premier livre ses pérégrinations à travers le monde, puis se concentre sur le royaume de France dans le deuxième livre, pour délivrer un manuel de bon gouvernement à l'usage du roi dans le troisième livre ${ }^{4}$.

Les figures allégoriques du Songe du vieil pelerin sont généralement des vertus morales ${ }^{5}$, mais la mise en récit se fonde également sur la construction d'un personnel ad hoc: Charles VI est notamment appelé le «Blanc faucon au bec et piez dorez» et le pape Urbain VI «le pasteur cornu». Les anecdotes édifiantes insérées dans le texte sont surtout empruntées aux biographies des Pères et des saints. Grand voyageur, Philippe de Mézières ne cesse en outre de faire référence à sa propre expérience ; il décrit par exemple certains aspects de la justice chypriote qu'il a connue au cours de ses voyages ${ }^{6}$. Tant du point de vue du personnel que des références convoquées, Le Songe du vieil pelerin semble se passer de mythes. Hésitant entre récit de voyage et construction allégorique autonome ${ }^{7}$, l'ouvrage ne semble pas a priori réinvestir de paradigmes mythiques.

${ }^{3}$ « Ardant Desir et sa suer Bonne Esperance sont prins en figure pour le Vieil Pelerin, aucteur de cestui livre, appelle songe ou vision, representans les personnes de tous ceulx qui desirent la reformacion de tout le monde et de toute la crestiente et par espicial du royaume de France $»$, ibid., p. 106.

${ }^{4}$ Joël Blanchard et Jean-Claude MüHLETHALER notent que le troisième livre du Songe du vieil pelerin est le seul de l'ouvrage que l'on puisse considérer comme un miroir des princes, in Écriture et pouvoir à l'aube des temps modernes, Paris, Presses Universitaires de France, « Perspectives littéraires », 2002, p. 27.

5 Il s'agit quelquefois de péchés; c'est le cas pour Orgueil, Avarice et Luxure qui se présentent à la cour papale dans le deuxième livre.

${ }^{6}$ PhiLIPPE DE MÉzIÈreS, Le Songe du Vieil Pelerin, op. cit., I, p. 486-487.

7 Armand Strubel relève cette tension entre création d'un monde autonome et récit de voyage : «Le texte hésite entre l'espace du récit de voyage et celui, désincarné, de l'itinéraire allégorique ; la référence permanente au témoignage oculaire tourne à la chronique $(. .)$.$» , in$ 
Destiné à parfaire l'éducation politique du jeune souverain, Le Songe $d u$ vieil pelerin dessine le portrait d'un roi idéal. Depuis l'Ancien Testament, la définition de la royauté prend le tyran pour contre-modèle récurrent et efficace. Dans le Deutéronome, une image optimiste de la personne du roi est esquissée ${ }^{8}$; en revanche, quand Yahvé répond au peuple qui lui demandait un roi, l'Ancien Testament en fait un inévitable tyran qui asservira les Hébreux ${ }^{9}$. Cette opposition parcourt tout le texte de Philippe de Mézières, qui n'hésite pas à faire allusion aux tyrans italiens dont il a fait l'expérience pour mettre en garde Charles VI, blanc faucon innocent qui «n'avoit encores mostre ne exerce aucune tyrannie », comme le rappelle le prologue ${ }^{10}$. L'élaboration de la figure royale se fait ainsi à l'ombre de celle du tyran.

Dans le troisième livre du Songe $d u$ vieil pelerin, la Reine Vérité entreprend l'éducation du jeune roi en prenant pour canevas un gigantesque échiquier moral. Ce long développement est introduit par le rappel des origines du jeu d'échecs. Philippe de Mézières emprunte alors la légende forgée par Jacques de Cessoles dans son De Ludo scacchorum, premier des ouvrages systématisant le recours allégorique à la grille de l'échiquier, rédigé au cours de la deuxième moitié du XIII ${ }^{\mathrm{e}}$ siècle et largement diffusé dès sa composition ${ }^{11}$. Selon ce frère dominicain, les échecs auraient été inventés par le philosophe

Armand StRUBEL, «Grant senefiance a»: Allégorie et littérature au Moyen Âge, Paris, Champion, 2002, p. 267.

${ }^{8}$ Deutéronome, XVII, 14-20.

9 Premier Livre de Samuel, VIII, 10-18. Voir à ce sujet l'article de Jacques LE GofF, «Portrait du roi idéal », in L'Histoire, n81, 1985, p. 70-76.

${ }^{10}$ PhILIPPE DE MÉZIÈRES, Le Songe du Vieil Pelerin, op. cit., I, p. 98.

${ }^{11}$ Pour le texte latin, voir l'édition d'Ernst KöPKE in Jahresbericht der Mittheilungen aus den Handschriften der Ritter-Akademie zu Brandenburg, II, Brandenburg, 1879. Pour une traduction médiévale, voir JACQUES DE CESSOLES, Le Jeu des eschaz moralisé, traduction de JeAn Ferron, édition publiée par Alain Collet, Paris, Honoré Champion, "Classiques français du Moyen Âge », 1999. Sur la diffusion du texte, voir notamment l'introduction d'Alain Collet à JACQUES DE CESSOLES, Le Jeu des eschaz moralisé, traduction de JEAN FERRON, op. cit., p. 84 sqq. 
Philometor afin de corriger le tyran Evilmerodag, roi de Babylone et fils de Nabuchodonosor $^{12}$. En lui apprenant les règles du jeu, le philosophe lui aurait donné des instructions pleines de sagesse et le tyran sanguinaire serait devenu un roi juste.

Parallèlement à l'inscription ponctuelle de cette légende dans le déroulement du récit, le troisième livre du Songe du vieil pelerin est celui qui fait le plus grand usage d'un autre paradigme préexistant pour modeler le portrait du souverain. Afin d'éduquer le jeune roi, Vérité lui fait parcourir en figure l'itinéraire de Moïse. Charles VI est ainsi appelé tout au long du troisième livre « jeune Moyse », modèle vétéro-testamentaire réinvesti pour forger l'image royale. L'objet de notre enquête est de comparer l'utilisation de ces deux paradigmes qui, s'ils ne relèvent pas du terreau de la mythologie antique, n'en demeurent pas moins mythiques, au sens de figures fondatrices, préexistant à la construction du récit, et marquées du triple sceau de l'ailleurs, de l'autrefois et de l'altérité. La construction textuelle d'un Charles VI rêvé se joue certes à l'ombre du tyran dans Le Songe du viel pelerin, mais se fonde également sur l'articulation de trois figures, celles du roi, du tyran et du sage.

\section{Le tyran et le sage}

Dans son De Ludo scacchorum, Jacques de Cessoles prend les échecs pour modèle d'une société idéale. En guise d'introduction à son traité, il relate les conditions d'invention du jeu et inscrit les échecs, encore en quête de reconnaissance au moment où il compose son recueil d'exempla, dans un temps long. Par cette fiction, le prédicateur dominicain érige les origines du jeu au rang

\footnotetext{
${ }^{12}$ Evil-Merodak est mentionné dans le Deuxième Livre des Rois, XXV, 27 et dans Jérémie, LII, 31, comme le roi de Babylone qui gracia Joiakîn, roi de Juda, le tira de prison et l'entretint toute sa vie durant. Il s'agit d'une figure à peine esquissée dans la Bible.
} 
de mythe et tente de légitimer le support ludique de son ouvrage édifiant. En prenant un échiquier pour canevas dans le troisième livre du Songe du vieil pelerin, Philippe de Mézières tient non seulement compte des goûts de Charles VI, qui jouait aux échecs ${ }^{13}$, mais fait également référence au traité de Jacques de Cessoles, dont plusieurs exemplaires figuraient dans la bibliothèque royale ${ }^{14}$. L'allégorie de l'échiquier est prétexte à des considérations politiques et morales dans Le Songe $d u$ vieil pelerin; elle semble moins motivée que dans le De Ludo scacchorum, où chacune des règles du jeu est associée à un ou plusieurs comportements sociaux. Pourquoi Philippe de Mézières, pour qui la grille de l'échiquier sert avant tout de grille ordonnatrice, a-t-il conservé ce récit des origines ?

Les bourdes des romans sont condamnées dans Le Songe du vieil pelerin, le narrateur les opposant à la vérité historique des chroniques ${ }^{15}$. Dans cette perspective, le statut de la légende est problématique puisque l'ouvrage de Jacques de Cessoles ne peut être érigé au rang d'auctoritas, à la différence de l'histoire antique ou des Écritures saintes. Si la référence intertextuelle est exhibée - le récit commence par « il fu ja dit » et ne cesse de renvoyer au « livre

13 « voyre affin que tu preignes plaisance ou dit livre qui est bon et bel et prouffitable a ta royale mageste, qui sces bien jouer aux eschez, affin que tu ente(n)s bien le mistere des traiz du roy, de la royne et des autres tes subgiez (...) », PhILIPPE DE MÉZIÈres, Le Songe du Vieil Pelerin, op. cit., II, p. 200-201. Outre cette remarque de l'auteur, on sait que Charles VI avait reçu à quinze ans « 2 tabliers de cipres ouvrez et garniz de tables et eschais pour l'esbatement du roi », Louis DouËT D’ARCQ, Comptes de l'Hôtel des Rois de France au XIV siècle et XV siècle, Paris, Renouard, 1865, p. 215.

${ }^{14}$ Voir Jean-Luc DeUfFiC (éd.), La Bibliothèque royale du Louvre, Le livre médiéval II, PECIA, 2004, par exemple p. 35 et 65.

15 «Et pource est il expedient, Beau Filz, pour ton govuernement, que tu (te) doyes garder de toy trop delicter es escriptures qui sont aprocrifes, et par espicial des livres et des romans qui sont rempliz de bourdes et qui attrayent le lysant souvent a impossibilite, a folie, vanite et pechie, comme les livres des bourdes de Lancelot et semblables (...), PHILIPPE DE MÉZIÈrES, Le Songe du Vieil Pelerin, op. cit., II, p. 220-221. 
des eschez ${ }^{16}-$ le nom de l'auteur initial n'est pas pour autant cité. Par ailleurs, Philippe de Mézières efface du récit toute référence précise : si Jacques de Cessoles nommait ses protagonistes et se livrait même à une étude onomastique $^{17}$, l'auteur du Songe du vieil pelerin se contente de laisser planer une indéfinition sur l'identité des personnages, appelés « un roy » et « ung saige philosophe $»^{18}$. De même, aucune indication n'est donnée sur le lieu supposé de l'invention - Babylone chez Jacques de Cessoles. La légende est certes conservée, mais l'altérité et l'ailleurs caractérisant le récit originel sont gommés. Le statut de cette légende est quasiment un hapax au sein de l'ouvrage où l'on trouve bien peu de récits aussi flous; des précisions concernant les protagonistes des exemples relatés sont toujours données, qu'il s'agisse d'épisodes bibliques ou d'histoires que l'auteur a lui-même entendues.

L'inscription masquée d'Evilmerodag au seuil de la longue allégorie de l'échiquier moral n'obéit pas aux mêmes impératifs que chez Jacques de Cessoles. Chez ce dernier, le récit des origines mythiques du jeu permettait de souligner l'intérêt politique des échecs. La fiction forgée par l'auteur met en effet en scène le problème du tyrannicide, très discuté à la fin du Moyen Âge. La solution proposée par le dominicain est douce, car c'est par l'intermédiaire du jeu que le philosophe corrige le roi ${ }^{19}$. La suite du traité s'emploie à retranscrire les vertus politiques des règles du jeu, tandis qu'elles ne sont pas évoquées chez Philippe de Mézières. La question de la tyrannie est pourtant au cœur du propos

\footnotetext{
${ }^{16}$ Ibid., II, p. 200.

17 « Le jeu trouva .I. philosophe d'Orient qui avoit nom Xerces en calde ou Philometor en grec, qui vault autant dire en françois comme cellui qui aime justice et mesure. », JACQUES DE CESSOLES, Le Jeu des eschaz moralisé, traduction de JEAN FERRON, édition publiée par Alain COLLET, op. cit., p. 129.

${ }^{18}$ PhilipPe De MÉzIÈRES, Le Songe du Vieil Pelerin, op. cit., II, p. 200.

19 Voir Raymond D. DI LoRENZO, «The Collection Form and the Art of Memory in the 'Libellus super ludo scacchorum' of Jacobus de Cessolis », in Mediaeval Studies, Pontifical Institue of Mediaeval Studies, Toronto, Canada, vol. XXXV, 1973, p. 208.
} 
de ce dernier, qui en avait fait l'expérience au cours de ses voyages. Le couple formé par le souverain et le sage dans la légende vient s'ajouter à une série de références précises renvoyant aux contingences actuelles ou à l'histoire récente dans le reste du Songe: de nombreuses anecdotes et digressions renvoient par exemple aux tyrans italiens contemporains de l'auteur ${ }^{20}$. L'utilisation du récit s'opère ainsi au profit d'un haut degré d'abstraction. Face au recours massif à l'actualité dans Le Songe du vieil pelerin, la légende tend davantage vers une généralisation du discours ${ }^{21}$. Les personnages d'Evilmerodag et de Philometor, dont les noms sont passés sous silence, deviennent emblématiques de ceux du tyran et du conseiller politique en général. L'altérité du mythe, en tant que récit qui se donne pour extérieur, est gommée pour en faciliter l'insertion dans le projet de l'auteur.

Les conditions de production du Songe du vieil pelerin réactivent par ailleurs un sens latent de la fiction. Miroir tendu vers Charles VI, l'ouvrage semble faciliter une lecture qui ferait du mythe des origines des échecs une mise en abyme du dessein politique de Philippe de Mézières. Le sage Philippe/Philometor conseillerait ainsi le souverain Charles VI/Evilmerodag et

${ }^{20}$ « Cy parle l'aucteur de la grande et merveilleuse tyrannie de cellui qui regne en la cite de Padue et des grans maulx qui en so(n)t advenuz, et puis des deux mastins de la cite de Veronne », PhILIPPE DE MÉZIÈRES, Le Songe du Vieil Pelerin, op. cit., I, p. 278.

${ }^{21}$ Sur l'oscillation entre effet référentiel et tendance au général, voir les remarques d'Armand STRUBEL dans « Le Songe du viel pelerin et les transformations de l'allégorie au quatorzième siècle », Perspectives médiévales, t. 6, 1980, p. 54-74. Voir également l'article de JeanClaude MÜHLETHALER, «Le tyran à table. Intertextualité et référence dans l'invective politique à l'époque de Charles VI », in Joël BLANCHARD (éd.), Représentation, pouvoir et royauté à la fin du Moyen Âge, Actes du colloque organisé par l'Université du Maine les 25 et 26 mars 1994, Paris, Picard Éditeur, 1995, p. 49-62 ainsi que les remarques de Jeannine QUiLlet sur le songe médiéval dans "IXe colloque (1973). Le songe », in Geneviève HASENOHR et Jean LONGÈRE (éd.), Culture et travail intellectuel dans l'occident médiéval, Paris, Centre national de la recherche scientifique, 1981, p. 81-93. 
l'auteur vient souligner ce parallèle ${ }^{22}$. Le récit inventé par Jacques de Cessoles met en lumière la nécessaire présence du sage dans la cité ${ }^{23}$. La position du philosophe/conseiller n'est pas sans rappeler la position occupée par Philippe de Mézières lui-même ${ }^{24}$. L'insistance plus ou moins explicite sur l'importance du sage au fil du Songe du vieil pelerin ${ }^{25}$ peut sans doute expliquer l'une des transformations que le précepteur de Charles VI fait subir au récit. Chez Jacques de Cessoles, le jeu est inventé par un philosophe et l'auteur du De Ludo scacchorum relate cette origine. En revanche, chez Philippe de Mézières, le philosophe et l'auteur du livre ne forment plus qu'un. Afin d'éduquer le roi, le philosophe lui présente le Livre des eschez ${ }^{26}$; auteur et sage sont donc confondus. Philippe semble conserver le récit des origines légué par la tradition textuelle en le remodelant afin de valoriser son propre rôle d'auteur/conseiller. Le roi peut se garder de tyrannie à condition d'être à l'écoute de ses conseillers. Le souverain idéal se construit certes à l'ombre de systèmes normatifs

\footnotetext{
${ }^{22}$ C'est la Reine Vérité qui, dans le dispositif narratif, est ici le porte-parole de l'auteur : “' A nostre propoz', dist la royne, 'le philosophe doncques presenta au dit roy le livre et le jeu des eschez pour le rappeler soutilement de sa grant tyrannie. Et je te presente a present le nouvel eschequier,' dist la royne, 'Beau Filz, pour toy garder de toute tyrannie. », PHILIPPE DE MÉzIÈres, Le Songe du Vieil Pelerin, op. cit., II, p. 201.

${ }^{23}$ Voir à ce sujet Jean-Michel MEHL, Jeu d'échecs et éducation au XIII siècle. Recherches sur le "Liber de moribus » de Jacques de Cessoles, Université des sciences humaines de Strasbourg, 1975, p. 183.

${ }^{24}$ Le personnage du conseiller royal est d'ailleurs présent dès le début de l'ouvrage puisque, avant d'entamer leur voyage à travers le monde, Ardant Désir et Bonne Espérance se rendent auprès de l'ermite Arsène. Or, saint Arsène fut le conseiller de l'empereur Théodose $\mathrm{I}^{\mathrm{er}}$ et le précepteur de ses fils. Cf. Dora M. BELl, Étude sur le Songe du Viel Pelerin, Genève, Droz, 1955, p. 22, note 7.

${ }^{25}$ Pour une réflexion sur l'importance des sages et des conseillers, voir le récit d'une nef aux prises avec une tempête : «Nul ne porroit', dist la chambriere, 'a plain reciter les maulx qui adviennent en une nef quant le patron ne gouverne pas par le conseil des saiges conseilliers, marchans et preudommes (...) », PHILIPPE DE MÉzIĖRES, Le Songe du Vieil Pelerin, op. cit., I, p. 583.

${ }^{26}$ « Le noble philosophe lors, quant il veit son point pour desclairer l'exposicion des eschez au dit roy, il presenta le livre des eschez. », ibid., II, p., 200.
} 
exemplaires ou contre-exemplaires, mais ne peut faire l'économie des préceptes du sage.

\section{Le tyran et le roi}

Evilmerodag emblématise la figure du tyran, contre-modèle récurrent des miroirs des princes. Les auteurs n'hésitent pas à brandir ce «sombre épouvantail », outil efficace de pédagogie politique ${ }^{27}$. L'antithèse parfaite du roi idéal s'assortit dans le troisième livre du Songe du vieil pelerin du recours à la figure positive de Moïse. La nature monarchique du pouvoir d'Israël ayant toujours été posée comme une évidence, le modèle du souverain biblique a souvent servi pour penser le prince médiéval ${ }^{28}$. Cependant, les auteurs des miroirs des princes carolingiens ont plutôt adopté pour modèles les rois de l'Ancien Testament, David et Salomon notamment. Il convient donc de se demander pourquoi Philippe de Mézières choisit d'associer le jeune Charles VI à ce personnage biblique ${ }^{29}$. Cette appellation entre en concurrence avec «le cerf

${ }^{27}$ Jacques KRYNEN, Idéal du prince et pouvoir royal en France à la fin du Moyen Âge, Paris, Éditions A. et J. Picard, 1981, p. 334.

${ }^{28}$ Voir sur ce point l'article de Rémi BRAGUE, «Du prince au peuple. La sagesse politique dans la Bible », in Ran HALÉVI (dir.), Le Savoir du prince du Moyen Âge aux Lumières, Paris, Fayard, « L'esprit de la cité », 2002, p. 7-23.

${ }^{29}$ C'est dans la bouche de Moïse que l'on trouve le code de bonne conduite du monarque dans le Deutéronome et c'est peut-être pour cette raison que ce personnage biblique sert à certains auteurs de modèle dans l'élaboration de figures royales idéales. On retrouve dans le choix de Philippe de Mézières un procédé déjà mis en œuvre par Eusèbe de Césarée qui, au début du $\mathrm{IV}^{\mathrm{e}}$ siècle, dans la Vie de Constantin, comparait certains épisodes de la vie de l'empereur à celle de Moïse, comme, par exemple, son enfance parmi les tyrans; voir Eusebius, Life of Constantine, Avril CAMERON et Stuart G. HALl (transl.), Oxford, Clarendon Press, 1999, p. 73. On trouve également des récits comparant Louis XI à Moïse, voir Edina Bozòky, La Politique des reliques de Constantin à saint Louis. Protection collective et légitimation du pouvoir, Paris, Beauchesne, 2006, p. 167. Quelques années après la rédaction du Songe du vieil pelerin, ChRISTINE DE PIZAN compare, ponctuellement cette fois, Charles V à un nouveau Moïse: "D'ycelle ditte noble lignée, Dieu ameur du tres crestien peuple françois, pour la reparacion, confort et preservacion du dit lieu (...), tout ainsi comme jadis donna Moyse, né de nobles parens, ou temps de l'adversité d'Egipte, aux enfens 
volant couronné », « le blanc faucon pelerin au bec et piez dorez », " le grand maistre du grant parc, jardriner du grant jardrin des blanches fleurs dorees », «le grant maistre des eauues et des forestz de France», ou encore «le grant marchant du grant change $»^{30}$. Afin de guider la lecture du jeune roi, l'auteur a doté son ouvrage d'une table des allégories : les équivalences sont explicitées au début du texte et le lecteur royal sait notamment qui le représentera dans la suite du récit ${ }^{31}$. Moïse est non seulement la seule figure biblique à apparaître dans cet outil, mais également le seul personnage déjà constitué.

Identifier Charles VI à Moïse, c'est tout d'abord dessiner un parcours. Le troisième livre du Songe du vieil pelerin décrit en effet l'ascension spirituelle du nouveau Moïse sur le Mont Sinaï, où, après avoir été éduqué par la Reine Vérité, qui inscrit les commandements de Dieu sur les tables de son cœur, il sera digne de recevoir les emblèmes de la royauté. Les épisodes de la vie de Moïse sont interprétés de façon allégorique. La Reine Vérité explique que Charles VI est un «Moyse couronné » car il fut «prins et extraiz des eauues d'Egypte, c'est assavoir du grant fleuve et riviere lacrimable des laermes qui furent espandues devant Dieu des bonnes personnes du peuple de France qui se doubtoient que par deffaulte de hoir masle, la couronne du royaume de Gaule n'yssist dehors de droicte ligne, par laque(lle) mutacion ou translacion et par adventure mauvais gouvernement (le royaume) n'alast a perdicion ${ }^{32} »$. Outre cette lecture

d'Israel, le sage conduiseur, pour le dit peuple en espace de jours tyrer hors du servage de Pharaon, voult la divine Providence faire naistre de parens solemnelz et dignes, c'est assavoir, du bel et chevalereulx Jehan, roy de France, et de la royne Bonne, s'espouse, fille du bon roy de Bahaigne, ycellui sage Charles, lequel fu le .LVI e roy de France, puis le roy Pharramont dit dessus regnans glorieusement par l'espace de mile XXIII ans courus jusques au couronnement d'ycellui dit sage roy Charles. ", Le Livre des fais et bonnes meurs du sage roy Charles $V$, Suzanne Solente (éd.), Paris, Honoré Champion, 1936, $1^{\mathrm{er}}$ volume, p. 14.

${ }^{30}$ PhilipPe DE MeZIERES, Le Songe du Vieil Pelerin, op. cit., I, p. 110.

${ }^{31}$ L'auteur l'intitule la « table figuree », « table des divers noms sus quoy est prinse la figure de l'entendement de cestui livre », ibid., I, p. 106.

${ }^{32}$ PhilipPe De MÉZIÈRES, Le Songe du Vieil Pelerin, op. cit., II, p. 136. 
allégorique des épisodes bibliques, Philippe de Mézières érige Moïse en modèle de comportement et de piété : la Reine Vérité rappelle à Charles VI qu'il « fu le plus debonnayre de tous les hommes qui lors estoient sus la terre $»^{33}$. La référence vétéro-testamentaire permet à la fois de structurer le troisième livre du Songe du vieil pelerin et d'offrir au roi un reflet normatif.

Le réinvestissement de ce personnage et des épisodes qui lui sont associés déborde cependant le cadre du paradigme du bon souverain. Faire de Charles VI un «nouveau Moïse », c'est faire du peuple du royaume de France un nouveau populus $d i^{34}$; le texte établit d'ailleurs explicitement cette comparaison ${ }^{35}$. Par la même occasion, le jeune roi est celui qui libèrera le peuple du joug qui l'opprime - la période de troubles politiques qui suit la mort de Charles V et précède l'accession au pouvoir de son fils. Les ambitions réformatrices de l'auteur se fondent sur l'espoir suscité par un Charles VI quasi-messianique, dont il dessine un portrait idéalisé. L'assimilation à Moïse permet ainsi de relire l'actualité à la lumière du mythe - au sens de paradigme déjà constitué extérieur à la fiction en construction - et de légitimer le pouvoir royal.

L'élaboration de la figure du destinataire trouve dans le personnage de Moïse un modèle efficace, parfaitement adaptable aux circonstances de production et de diffusion de l'ouvrage. Par ailleurs, l'inscription de ce personnage biblique est en relation avec l'omniprésence de la figure du tyran. On a en effet vu dans l'épisode de l'Exode où Moïse tue un Égyptien, qui avait

\footnotetext{
${ }^{33}$ Ibid., II, p. 162.

${ }^{34}$ Voir Yves SASSIER, Royauté et idéologie au Moyen Âge. Bas-Empire, monde franc, France $\left(I V^{e}-X I I^{e}\right.$ siècle), Paris, Armand Colin, 2002, p. 39 à propos de 1'Empire romain.

35 «Moyse, souverain prince d'Israel, c'est assavoir Charles $\mathrm{VI}^{\mathrm{e}}$ de ce nom, souverain prince, empereur et roy de la generacion gallicane, laquelle peust bien estre dicte peuple d'Israel, c'est assavoir veoiant Dieu par la foy catholique », PHILIPPE DE MÉZIÈRES, Le Songe du Vieil Pelerin, op. cit., I, p. 473.
} 
lui-même tué un Israélite ${ }^{36}$, l'une des premières justifications du tyrannicide ${ }^{37}$.

Charles VI, roi idéal rêvé par Philippe de Mézières, est un souverain fermement opposé à toute forme de tyrannie et Moïse en est ainsi l'un des avatars possibles.

\section{Le roi et le sage}

Le choix de Moïse permet de brosser un portrait royal treschretien, conformément aux desseins de l'auteur qui appelle à une reformacion de la Chrétienté $^{38}$. Toutefois, Moïse peut également apparaître comme une figure de messager privilégié, ou encore d'auteur. Le Songe du vieil pelerin joue d'ailleurs à plusieurs reprises de cette ambiguiité. Ardant Désir, que la table des allégories au début de l'ouvrage associe explicitement à l'auteur, se compare à Moïse en raison de son bégaiement ${ }^{39}$. Philippe de Mézières lui-même s'est à plusieurs reprises comparé à ce personnage. Lors d'un pèlerinage à Jérusalem, dans l'église du Saint-Sépulcre, il eut une vision du Christ qui lui donna les Tables de la Loi $^{40}$. En 1384, soit cinq ans avant la fin de la rédaction du Songe du vieil pelerin, il adresse un appel à la chrétienté depuis le couvent des Célestins. Dieu lui inspire alors deux nouvelles Tables de la Loi $^{41}$. Le paradigme choisi pour

\footnotetext{
${ }^{36}$ Exode, II, 12.

${ }^{37}$ C'est notamment le cas de Jean Petit, maître de l'Université de Paris, qui au début du XV siècle s'efforce de justifier le duc de Bourgogne, Jean sans Peur, de l'assassinat du duc Louis d'Orléans, frère de Charles VI. Voir Alfred CoviLle, Jean Petit. La question du tyrannicide au commencement du XV $V^{e}$ siècle, Genève, Slatkine Reprints, 1974 ( $1^{\text {ère }}$ édition, 1932), p. 180.

38 Dans la perspective idéologique de Philippe de Mézières, il peut sembler étonnant que l'épisode du passage de la mer Rouge ne fasse pas l'objet d'une lecture typologique, à la lumière de son appel à la reprise des croisades.

${ }^{39}$ Ardant Desir, répondant à Divine Providence, affirme dès le prologue : «je ne suis pas digne d'une si grant legation ne d'une si grant messagerie, pource que avecques Moyse je suis begue et ne scay pas bien parler », PHILIPPE DE MÉzIÈRES, Le Songe du Vieil Pelerin, op. cit., I, p. 95 ; voir aussi I, p. 366.

${ }^{40}$ Nicolas JoRgA, Philippe de Mézières (1327-1405) et la croisade au XIV siècle, Genève, Slatkine reprints, Paris, Honoré Champion, 1976, $\left(1^{\text {ère }}\right.$ édition Paris, Librairie Émile Bouillon, 1896), p. 73.

${ }^{41}$ Ibid., p. 453-454.
} 
dessiner un roi idéal dans Le Songe du vieil pelerin est donc un modèle qui obsédait l'auteur ${ }^{42}$.

Non seulement l'incarnation narrative de Charles VI par Moïse sacralise le corps du roi, directement en contact avec Dieu, mais elle joue également d'une certaine ambiguité liée au statut de ce personnage chez Philippe de Mézières. En établissant des comparaisons entre les figures de l'auteur et l'une des figures incarnant le destinataire royal dans le récit allégorique, le précepteur de Charles VI brouille les pistes. Un même référent est associé au roi et au sage au sein du Songe du vieil pelerin. Dans un article consacré aux songes politiques au Moyen Âge, Christiane Marchello-Nizia remarque que si d'ordinaire dans les songes, le rêveur est concerné au premier chef par ce qu'il voit, il en est tout autrement des songes politiques. Le rêveur y est le siège d'une vision qui concerne le pouvoir. Or, «se donner comme le siège d'un rêve royal, comme le détenteur d'une connaissance politique révélée, c'est usurper une fonction royale $^{43} \gg$. C'est pour cette raison que ces songes mettent généralement en œuvre des stratégies de brouillage de l'instance énonciative, dont la référence à Moïse semble être l'une des manifestations dans Le Songe du vieil pelerin. En comparant ses doubles allégoriques au personnage incarnant narrativement le souverain, l'auteur s'érige subtilement en figure royale, tout en neutralisant ce désir, puisqu'il ne s'agit que d'un songe.

Evilmerodag et Moïse sont donc deux personnages que tout semble opposer et dont Philippe de Mézières se sert pour modeler, entre idéal et contre-

\footnotetext{
42 Joël Blanchard, «Politique des points de vue et stratégies discursives. Philippe de Mézières et le discours de la réformation morale ", in Actes du $18^{e}$ Congrès International de Linguistique et de Philologie Romanes, Trèves, 1986, t. 6, p. 498.

43 Christiane MARChEllo-NizIA, «Entre l'histoire et la poétique, le Songe politique », in Revue des Sciences humaines, t. 183, 1981, "Moyen Âge flamboyant: XIV $-\mathrm{XV}^{\mathrm{e}}$ siècles », p. 53.
} 
exemple, le portrait du bon roi. Prophète en relation directe avec Dieu et libérant le peuple, Moïse est en effet bien loin du sanguinaire Evilmerodag. Ces deux paradigmes s'inscrivent dans l'ouvrage de Philippe de Mézières de façon distincte. La légende du tyran de Babylone corrigé par le philosophe occupe un espace narratif défini ; le modèle de Moïse est sur-représenté, surtout dans le troisième livre du Songe $d u$ vieil pelerin, sous la forme d'un paradigme structurant le récit et d'adresses récurrentes au destinataire. Malgré des différences de traitement, Evilmerodag et Moïse ont en commun d'être liés à des récits fondateurs, l'un relatant l'invention des échecs, l'autre les origines de toute la tradition chrétienne. Ces deux références dressent ainsi le portrait, en creux ou positivement, d'un roi hors-temps ${ }^{44}$. Il s'agit d'inscrire le temps biographique de Charles VI dans un temps mythique, celui des origines.

L'élaboration de la figure du roi idéal dans Le Songe du vieil pelerin ne se fait pas uniquement à l'ombre du contre-exemple tyrannique. Afin d'éviter toute forme de tyrannie, le bon souverain doit en effet être à l'écoute des sages. L'utopie d'un bon gouvernement selon Philippe de Mézières prend en compte le conseiller, position qu'il occupait lui-même. En offrant au jeune roi un miroir des princes, l'auteur semble parfois se perdre dans la contemplation de sa propre image.

\footnotetext{
${ }^{44}$ Voir à ce sujet les remarques de Giovanna ANGELI dans « Temps et histoire chez Christine de Pizan: Le Livre des fais et bonnes meurs du sage roy Charles $V$ », in Emmanuèle Baumgartner et Laurence Harf-LAnCNER (dir.), Dire et penser le temps au Moyen Âge. Frontières de l'histoire et du roman, Paris, Presses Sorbonne Nouvelle, 2005, p. 151-168.
} 